\title{
Glutaminyl cyclase activity correlates with levels of $A \beta$ peptides and mediators of angiogenesis in cerebrospinal fluid of Alzheimer's disease patients
}

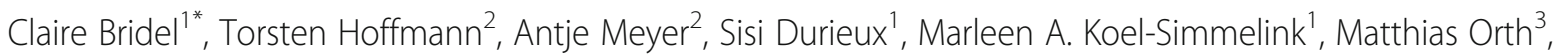
Philip Scheltens ${ }^{4}$, Inge Lues ${ }^{2}$ and Charlotte E. Teunissen ${ }^{1 *}$

\begin{abstract}
Background: Pyroglutamylation of truncated $A \beta$ peptides, which is catalysed by enzyme glutaminyl cyclase (QC), generates $p E-A \beta$ species with enhanced aggregation propensities and resistance to most amino-peptidases and endo-peptidases. $\mathrm{pE}-\mathrm{A} \beta$ species have been identified as major constituents of $A \beta$ plaques and reduction of $p E-A \beta$ species is associated with improvement of cognitive tasks in animal models of Alzheimer's disease (AD).

Pharmacological inhibition of QC has thus emerged as a promising therapeutic approach for AD. Here, we question whether cerebrospinal fluid (CSF) QC enzymatic activity differs between AD patients and controls and whether inflammatory or angiogenesis mediators, some of which are potential QC substrates, and/or A $\beta$ peptides may serve as pharmacodynamic read-outs for QC inhibition.

Methods: QC activity, A $\beta$ peptides and inflammatory or angiogenesis mediators were measured in CSF of a clinically well-characterized cohort of 20 mild AD patients, 20 moderate AD patients and 20 subjective memory complaints (SMC) controls. Correlation of these parameters with core diagnostic CSF AD biomarkers (Aß42, tau and p-tau) and clinical features was evaluated.

Results: $Q C$ activity shows a tendency to decrease with $A D$ progression $(p=0.129)$. The addition of $Q C$ activity to biomarkers tau and p-tau significantly increases diagnostic power (ROC-AUC $C_{\text {TAU }}=0.878, R O C-A \cup C_{T A U} \&_{O C}=0.939$ and ROC-AUC $\left.C_{\text {pTAU }}=0.820, R O C-A \cup C_{\text {pTAU }} \&_{Q C}=0.948\right)$. In AD and controls, $Q C$ activity correlates with $A \beta 38(r=0.83$, $p<0.0001)$ and A $340(r=0.84, p<0.0001)$, angiogenesis mediators (Flt1, Tie2, VEGFD, VCAM-1 and ICAM-1, $r>0.5$, $p<0.0001)$ and core diagnostic biomarkers $(r>0.35, p=<0.0057)$. QC activity does not correlate with MMSE or ApoE genotype.

Conclusions: $A \beta 38, A \beta 40$ and angiogenesis mediators (Flt1, Tie2, VEGFD, VCAM-1 and ICAM-1) are potential pharmacodynamic markers of QC inhibition, because their levels closely correlate with QC activity in AD patients. The addition of QC activity to core diagnostic CSF biomarkers may be of specific interest in clinical cases with discordant imaging and biochemical biomarker results.
\end{abstract}

Keywords: Alzheimer's disease, Cerebrospinal fluid, Glutaminyl cyclase, Amyloid beta, 3pE-Aß42

\footnotetext{
* Correspondence: c.bridel@vumc.nl; c.teunissen@vumc.nl

${ }^{1}$ Neurochemistry Lab and Biobank, Department of Clinical Chemistry, VU

University Medical Centre Amsterdam, Amsterdam, The Netherlands

Full list of author information is available at the end of the article
} 


\section{Background}

Soluble, non-fibrillar amyloid-beta $(\mathrm{A} \beta)$ oligomers are key players in the initiation and propagation of Alzheimer's disease (AD) pathology [1]. Variable amyloid precursor protein (APP) cleavage by $\beta$-secretases and $\gamma$-secretases generates a diversity of $A \beta$ species spanning 34-50 amino acids in length $[2,3]$. Further variability arises from $\mathrm{N}$ terminal truncations of these peptides $[4,5]$, and from N-terminal post-translational modifications including pyroglutamylation, isomerization and racemization [2]. Pyroglutamylation, a dehydration reaction converting $\mathrm{N}$-terminal glutamate $(\mathrm{E})$ into pyroglutamate $(\mathrm{pE})$, generates $\mathrm{pE}-\mathrm{A} \beta$ species with enhanced aggregation propensities [6-8] and resistance to most amino-peptidases and endo-peptidases [9]. pE-A $\beta$ species have been identified as major constituents of $A \beta$ plaques [10-12]. One of these, $3 \mathrm{pE}-\mathrm{A} \beta 42$, has gained much attention over the past years since the demonstration of its ability to trigger neurodegeneration when expressed transgenically in mouse [13] and to seed aggregation of $A \beta$ species to form highly toxic $\mathrm{A} \beta$ oligomers both in vitro and in vivo [14].

Pyroglutamylation of truncated $A \beta$ species carrying a glutamate residue at position 3 or 11 (including $A \beta 3-40$, $A \beta 11-40, A \beta 3-42$ and $A \beta 11-42)$ is catalysed by enzyme glutaminyl cyclase (QC) [15-17], whose expression is increased in temporal and enthorinal cortices of $\mathrm{AD}$ patients and correlates with insoluble $\mathrm{pE}-\mathrm{A} \beta$ aggregates [18]. In addition to $N$-terminal glutamate $A \beta$ species, QC catalyses pyroglutamation of a number of other substrates harbouring an $\mathrm{N}$-terminal glutamine residue, including inflammatory mediators monocyte chemoattractant proteins (MCP) 1-4 (also known as CCL2, CCL8, CCL7 and CCL13) [19] and possibly fractalkine (CX3CL1) (Kehlen et al., manuscript in preparation), C-reactive protein (CRP) [20] and soluble ICAM-1 [19, 21]. These may be of particular relevance to AD pathomechanisms, because local chronic inflammation resulting from microglia and astrocyte activation by $A \beta$ aggregates is a well-described feature of AD pathology [22-24] and affects APP and A $\beta$ processing [25-27].

In mouse, chronic pharmacological inhibition or genetic ablation of $\mathrm{QC}$ results in reduced $\mathrm{pE}-\mathrm{A} \beta$ brain levels and improvement of cognitive tasks in models of $\mathrm{AD}$ [16]. Reduction of $\mathrm{pE}-\mathrm{A} \beta$ levels through pharmacological inhibition of QC has thus emerged as a promising therapeutic approach for AD. QC inhibitor PQ912 was reported safe and well tolerated in a recent phase 1 study [28]. In young (18-50 years old) healthy individuals, PQ912 reduced both serum and cerebrospinal fluid (CSF) QC activity in a dose-dependent manner [28]. In the present study, we first measured QC enzymatic activity in CSF of AD patients and questioned whether it differed from that of age-matched controls, offering a prospect as a diagnostic or disease progression biomarker. Second, we set out to measure CSF levels of $\mathrm{A} \beta$ peptides, as well as angiogenesis and inflammatory mediators, whose levels may be modified in AD CSF, given the involvement of angiogenesis and inflammation in AD pathomechanisms [24, 29]. All parameters were analysed for differences between diagnosis groups and correlation with QC activity.

\section{Methods \\ Patients}

All patients and corresponding CSF samples were selected from the Alzheimer Center Memory Cohort, NeuroUnit Biomarkers for Inflammation and Neurodegeneration, VU Medical Center (VUmc) Biobank (Amsterdam, the Netherlands). We included 40 patients with probable AD and 20 patients with subjective memory complaints (SMC), which served as a control group. All patients underwent extensive dementia screening at baseline, including physical and neurological examination, EEG, MRI and laboratory tests. Neuropsychological assessment was performed and included the Mini Mental State Examination (MMSE) for global cognition. Diagnoses were made by consensus in a multidisciplinary meeting. Probable AD was diagnosed according to the core clinical National Institute on Aging-Alzheimer's Association (NIA-AA) criteria [30]. Mild AD $(n=20)$ is defined here as patients with MMSE score $>18$ and $<24$, and moderate AD $(n=$ $20)$ as patients with MMSE score $\leq 18$ and $\geq 15$. The cohort selection was based on short CSF storage time to avoid storage artefacts (mean storage time \pm SEM: $2.92 \pm$ 1.45 years) and MMSE score. Core CSF diagnostic biomarkers $(A \beta 42$, tau and $p$-tau) were not part of the diagnostic workup. Diagnosis of SMC was determined when results of all clinical examinations were normal, and there was no psychiatric diagnosis. The study was approved by the local ethical review board and all subjects gave written informed consent for the use of their clinical data for research purposes.

\section{CSF collection}

CSF was obtained by lumbar puncture using a 25-gauge needle, and collected in 10-ml polypropylene tubes (Sarstedt, Nümbrecht, Germany). Within 2 hours of collection, CSF was centrifuged at $1800 \times g$ for $10 \mathrm{~min}$ at $4{ }^{\circ} \mathrm{C}$, transferred to new polypropylene tubes and stored at $-80{ }^{\circ} \mathrm{C}$ until further analysis [31]. CSF was analysed without knowledge of clinical diagnoses.

\section{Core CSF diagnostic AD biomarkers}

Core diagnostic AD biomarkers tau and p-tau were measured in CSF by commercially available ELISAs (Innotest hTAU-Ag and Innotest Phosphotau (181P); Innogenetics, Ghent, Belgium), according to the manufacturer's instructions at the Neurochemistry Laboratory, VUmc. Core 
diagnostic $\mathrm{AD}$ biomarker $\mathrm{A} \beta \mathrm{x}-42$ as well as C-terminally truncated $A \beta$ variants $A \beta x-38$ and $A \beta x-40$ were measured using two commercially available multiplex immunoassays (Abeta 3-Plex Kit 6E10 and 4G8; Meso Scale Diagnostics, Rockville, USA). The first multiplex assay makes use of monoclonal antibody 6E10 (Covance; reactive to A $\beta 1-16$, binding epitope between amino acids 4 and 9) as the capture antibody, and peptide-specific detection antibodies. The second multiplex assay makes use of monoclonal antibody 4G8 (Covance; reactive to amino acids 17-24 of $A \beta)$ as the capture antibody, and peptide-specific detection antibodies. Method correlation between the 4G8 multiplex immunoassay and the 6E10 multiplex immunoassay was strong (see Additional file 1: Figure S2).

\section{QC activity in CSF}

QC activity in CSF was measured as described previously $[28,32]$ by a flourimetric assay using Gln-AMC as the substrate and pyroglutamyl aminopeptidase as the auxiliary enzyme, and was performed by Evotec AG (Hamburg, Germany).

\section{Truncated $A \beta$ peptides in CSF}

$N$-terminal variants of $A \beta$ peptides $A \beta 3-40, A \beta 11-40$ and A $\beta 11-42$ were quantified by liquid chromatography tandem mass spectrometry (LC-MS/MS). A suitable LC-MS/ MS approach for absolute quantification of $A \beta 1-40$ and A $\beta 1-42$ was described previously [33] and is currently under validation as a standard reference method [34, 35]. Method correlation between the multiplex immunoassays and LC-MS/MS for A $\beta$ quantification is shown in Additional file 2: Figure S3. We applied and further adapted this analytical approach by including also the $\mathrm{N}$-terminally truncated peptides $A \beta 3-40, A \beta 11-40, A \beta 3-42$ and $A \beta 11-$ 42. In contrast to Lame et al. [33], and due to the much lower abundance of the N-terminally truncated peptides, we used immuno-affinity enrichment instead of solidphase extraction to extract the peptides from CSF.

A previously described method by Kleinschmidt et al. [36] for $A \beta$ peptide extraction from human plasma using a mixture of three anti-A $\beta$ monoclonal antibodies was modified for the extraction of $100 \mu \mathrm{l} \mathrm{CSF}$. Equal aliquots of 4G8, 12 F4 and 11A50-B10 antibodies (2 $\mu$ g; supplied by Hiss Diagnostics, Germany) and stable isotopelabelled internal standards $(1 \mathrm{ng} / \mathrm{ml}$ final concentration, Additional file 3: Table S1) were added to $100 \mu \mathrm{l} \mathrm{CSF}$ in $400 \mu \mathrm{l}$ phosphate-buffered saline (PBS) containing $1 \%$ BSA and $0.05 \%$ Tween. After an overnight incubation on a rotary shaker at $4{ }^{\circ} \mathrm{C}$, the immuno-complex was coupled to pre-washed Dynabeads ${ }^{\mathrm{Tm}} \mathrm{M}-280$ Sheep AntiMouse IgG (Invitrogen) for another 2 hours at $25{ }^{\circ} \mathrm{C}$. The beads were separated magnetically, washed with PBS and twice with $25 \mathrm{mM}$ ammonium bicarbonate containing $0.1 \%$ octyl $\beta$-D-glucopyranoside and the peptides of interest were eluted using $100 \mu \mathrm{l}$ of $50 \%$ acetonitrile containing $1 \%$ ammonium hydroxide (50/50, $\mathrm{v} / \mathrm{v})$. Then $20-\mu \mathrm{l}$ extracts were injected into an XBridge BEH130 C18 column ( $3.5 \mu \mathrm{m}, 2.1 \mathrm{~mm} \times 150 \mathrm{~mm}$; Waters) operating at $50{ }^{\circ} \mathrm{C}$ using an UltiMate 3000 RSLC nano system (Dionex) (for a chromatogram of a sample extract, see Additional file 4: Figure S1). The mobile phase consisted of $0.1 \%$ ammonium hydroxide/acteonitrile $(95 / 5, \mathrm{v} / \mathrm{v}$ ) (solvent A) and $0.1 \%$ ammonium hydroxide $(5 / 95, \mathrm{v} / \mathrm{v})$ (solvent B). A linear gradient at a flow rate of $400 \mu \mathrm{l} / \mathrm{min}$ starting from $2 \%$ solvent B to $70 \%$ solvent B was applied for 3.5 min with a further increase to $100 \% \mathrm{~B}$ before re-equilibration. The LC was coupled to a TSQ Quantiva Triple Quadrupole mass spectrometer (Thermo Fisher Scientific) operating in selected reaction monitoring (SRM) positive mode using heated electrospray ionization.

Because absolute quantification depends on the quality of the reference items, we and others [35] discovered that determining the exact peptide content of $A \beta$ peptides appears to be difficult, due to potential chemical modifications, non-specific binding and a tendency for aggregation. Therefore, a relative quantification approach, considering the relative response of analyte versus internal standard, was used within this study. Nevertheless, calibration curves from spike-in of reference peptides into artificial CSF were prepared for each analytical batch and found to be linear within the anticipated concentration range. Reference peptides were synthesized by JPT Peptide Technologies GmbH (Germany) and Innovagen (Sweden). To assess the inter-assay variability, an endogenous CSF pool was prepared and spiked with internal standard peptides, stored at $-80{ }^{\circ} \mathrm{C}$ and analysed as a quality control sample within each analytical batch. The respective response ratios were stable over a month's storage (Additional file 2: Table S2).

\section{Inflammatory mediators}

CSF levels of inflammatory mediators were assessed using commercially available 37-plex ELISAs (Mesoscale Discovery, MSD) according to the manufacturer's instructions, which measure panels of proinflammatory molecules (IFN- $\gamma$, IL-1 $\beta$, IL-2, IL-4, IL-6, IL-8, IL-10, IL13, TNF $\alpha$ ), chemokines (Eotaxin, Eotaxin-3, IP10, MCP1, MCP-4, MDC, MIP- $1 \alpha$, MIP-1 $\beta$, TARC), cytokines (IL1- $\alpha$, IL-5, IL-7, IL-12, IL-15, IL-16, IL-17A, TNF- $\beta$, VEGF), angiogenesis mediators (bFGF, Flt1, PlGF, Tie2, VEGF-D, VEGF-C) and vascular injury markers (SAA, CRP, VCAM-1, ICAM-1).

\section{Statistical analysis}

$\mathrm{R}$ version 3.0.1, including packages "pgirmes", "pROC" and "ellipse", were used for statistical analysis and figure preparation. All biochemical CSF markers which were not normally distributed (Shapiro-Wilk test) were log- 
Table 1 Demographics and ApoE genotype proportion of cohort

\begin{tabular}{|c|c|c|c|c|c|c|c|}
\hline \multirow[t]{2}{*}{ Diagnosis } & \multirow[t]{2}{*}{$N$} & \multirow[t]{2}{*}{$\%$ female } & \multirow{2}{*}{$\begin{array}{l}\text { Mean (SD) } \\
\text { age (years) }\end{array}$} & \multirow{2}{*}{$\begin{array}{l}\text { Mean (SD) } \\
\text { MMSE score }\end{array}$} & \multicolumn{3}{|c|}{ ApoE genotype, $N(\%)$} \\
\hline & & & & & E3E3 & E3E4 & E4E4 \\
\hline Mild AD & 20 & 35.7 & $60.4(2.9)$ & $21.1(1.0)$ & $9(45 \%)$ & $10(50 \%)$ & $1(5 \%)$ \\
\hline Moderate AD & 20 & 61.5 & $60.1(2.8)$ & $15.1(2.0)$ & $5(25 \%)$ & $12(60 \%)$ & $3(15 \%)$ \\
\hline SMC & 20 & 42.0 & $60.3(2.7)$ & $26.9(1.9)$ & $9(47 \%)$ & $8(42 \%)$ & $2(11 \%)$ \\
\hline
\end{tabular}

AD Alzheimer's disease, SMC subjective memory complaints, MMSE Mini Mental Status Evaluation, ApoE apolipoprotein $\mathrm{E}$

transformed before correlation analyses to achieve normal distribution. Pearson's correlation coefficient $(r)$ and the respective $p$ values are provided in the figures. Groups were compared for all variables using the Kruskal-Wallis test. For each parameter, correction for multiple comparisons between groups was done using the function "kruskalmc" ("pgirmess" package) after the Kruskal-Wallis test. $p<0.05$ was considered significant. Because of the exploratory character of our study, no further correction for multiple testing was performed.

\section{Results}

Baseline characteristics and apolipoprotein E (ApoE) genotype distribution of the study population are presented in Table 1. Age was comparable between groups. E4/E4 and
E3/E4 genotypes were slightly more frequent in mild $A D$, but Fisher's exact test revealed no significant differences in ApoE genotype distribution between groups $(p=0.55)$.

\section{Levels of core diagnostic AD biomarkers, C-terminally} truncated $A \beta$ variants and $Q C$ activity in CSF of $A D$ patients Core diagnostic AD CSF biomarker (A $\beta 42$, tau and $\mathrm{p}$-tau) levels were distributed as expected across the three diagnosis groups: significantly lower levels of $A \beta 42$ in $A D$ patients and significantly higher levels of tau and p-tau in $\mathrm{AD}$ patients compared with SMC controls (Fig. 1c-e). Cterminally truncated $A \beta$ variants $A \beta 38$ and $A \beta 40$ showed similar trends towards decrease with progression of $\mathrm{AD}$, reaching significance between moderate $\mathrm{AD}$ and controls for A $\beta 40$ only $(p<0.05$, Fig. 1a, b). QC activity showed nominally decreased mean activities with $\mathrm{AD}$ progression, but

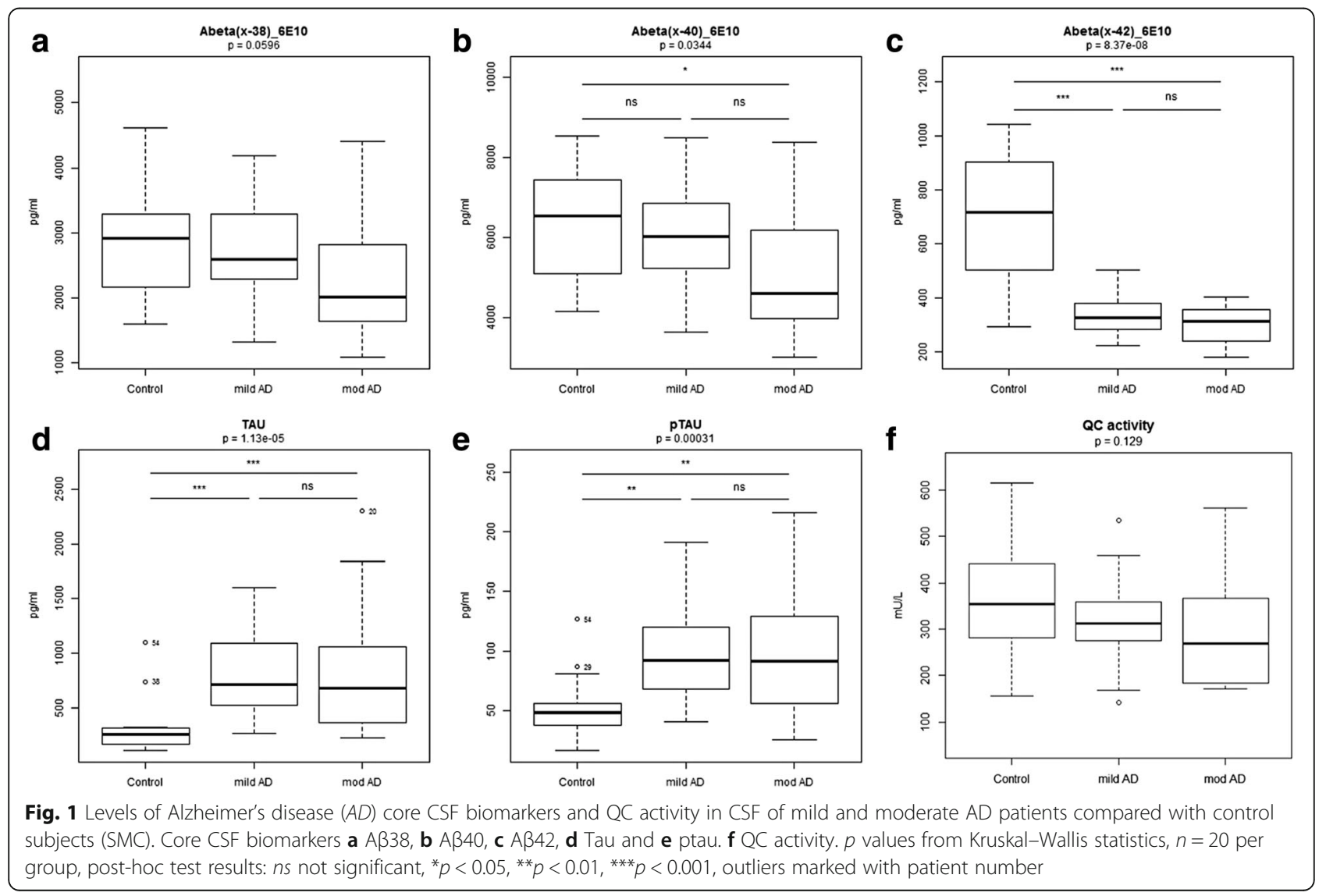


without reaching significance ( $p=0.129$, Fig. 1f). In CSF of $\mathrm{AD}$ and controls, LC-MS/MS allowed relative quantification of three $\mathrm{N}$-terminal-variants $\mathrm{A} \beta 3-40, \mathrm{~A} \beta 11-40$ and $\mathrm{A} \beta 11-42$, in addition to full-length peptides $A \beta 1-40$ and $A \beta 1-42$. These truncated peptides all carry an $\mathrm{N}$-terminal glutamate and are proven QC substrates [15, 17]. A $33-40$ showed a slight decrease of mean levels with progression of $\mathrm{AD}$, without reaching significance $(p=0.164)$ (Fig. 2a, b). A similar trend was observed for $\mathrm{A} \beta 1-40$ ( $p=$ 0.052). A $\beta 11-40(p=0.663)$ and A $\beta 11-42(p=0.068)$ levels showed no differences between diagnosis groups. A $\beta 11-42$ thus clearly differed from $A \beta 42$, whose levels differentiate controls and $\mathrm{AD}$ patients $(p<0.0001$, Fig. $2 \mathrm{c}-\mathrm{e})$. Levels of $\mathrm{A} \beta 3-42$ and $\mathrm{pE}$ modified species could not be detected with the LC-MS/MS method used in this study. Levels of full-length $A \beta 1-40$ and $A \beta 1-42$ determined by LC-MS/MS correlated well with respective $\mathrm{A} \beta 40$ and $\mathrm{A} \beta 42$ levels determined by ELISA $\left(r_{\mathrm{A} \beta 40}=0.944\right.$ and $r_{\mathrm{A} \beta 42}=0.933$, see Additional file 1: Figure S2).

\section{Correlation of QC activity with core AD biomarkers}

A high correlation of QC activity was observed with $A \beta 38$ and $\mathrm{A} \beta 40$, with Pearson correlation coefficients of 0.83 $(p<0.0001)$ and $0.84(p<0.0001)$ respectively (Fig. 3a, b). When considering all study subjects together, the correlations of QC activity with $\mathrm{A} \beta 42$, tau and p-tau were moderate, with correlation coefficients below 0.5 (Fig. 3ce, black lines). However, when evaluating each diagnosis group separately, we observed higher correlations of QC activity with $\mathrm{A} \beta 42$ in the control group $(r=0.56)$ and mild AD group $(r=0.66)$ and with tau and p-tau for both $\mathrm{AD}$ groups ( $r=$ up to 0.77 for QC-ptau correlation in moderate $\mathrm{AD}$ ) (Fig. 3c-e, coloured lines). Most strikingly, for tau and p-tau (Fig. 3d, e) higher correlations as well as a larger increase of tau respective to $\mathrm{p}$-tau per increased unit of QC activity were observed in the AD groups (Fig. 3d, e, blue and red lines) compared with the control group (green lines). This was reflected in a better diagnostic performance of a combination of tau and QC or p-tau and QC compared with tau or p-tau alone. Figure 4 shows logistic regression and receiver operating characteristic (ROC) curves for tau and p-tau and their combination with QC activity. With addition of the QC parameter, the area under the curve (AUC) increases from 87.8 to $93.9 \%$ $(p=0.032)$ and from 82.0 to $94.8 \%(p=0.002)$ for tau and p-tau respectively.

\section{Correlation of QC activity with inflammatory parameters}

A positive correlation was observed between QC activity and a number of analytes from the angiogenesis panel.

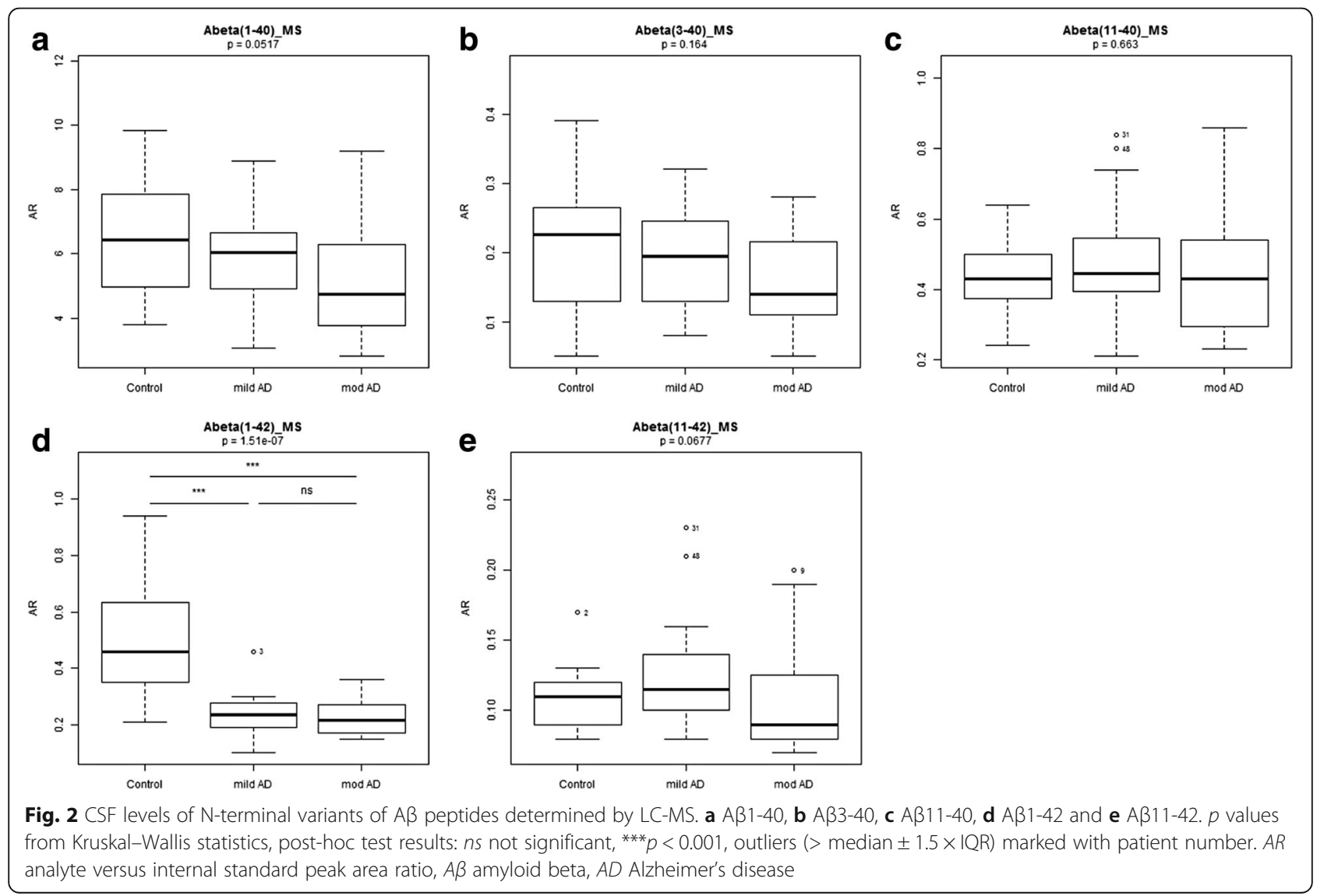



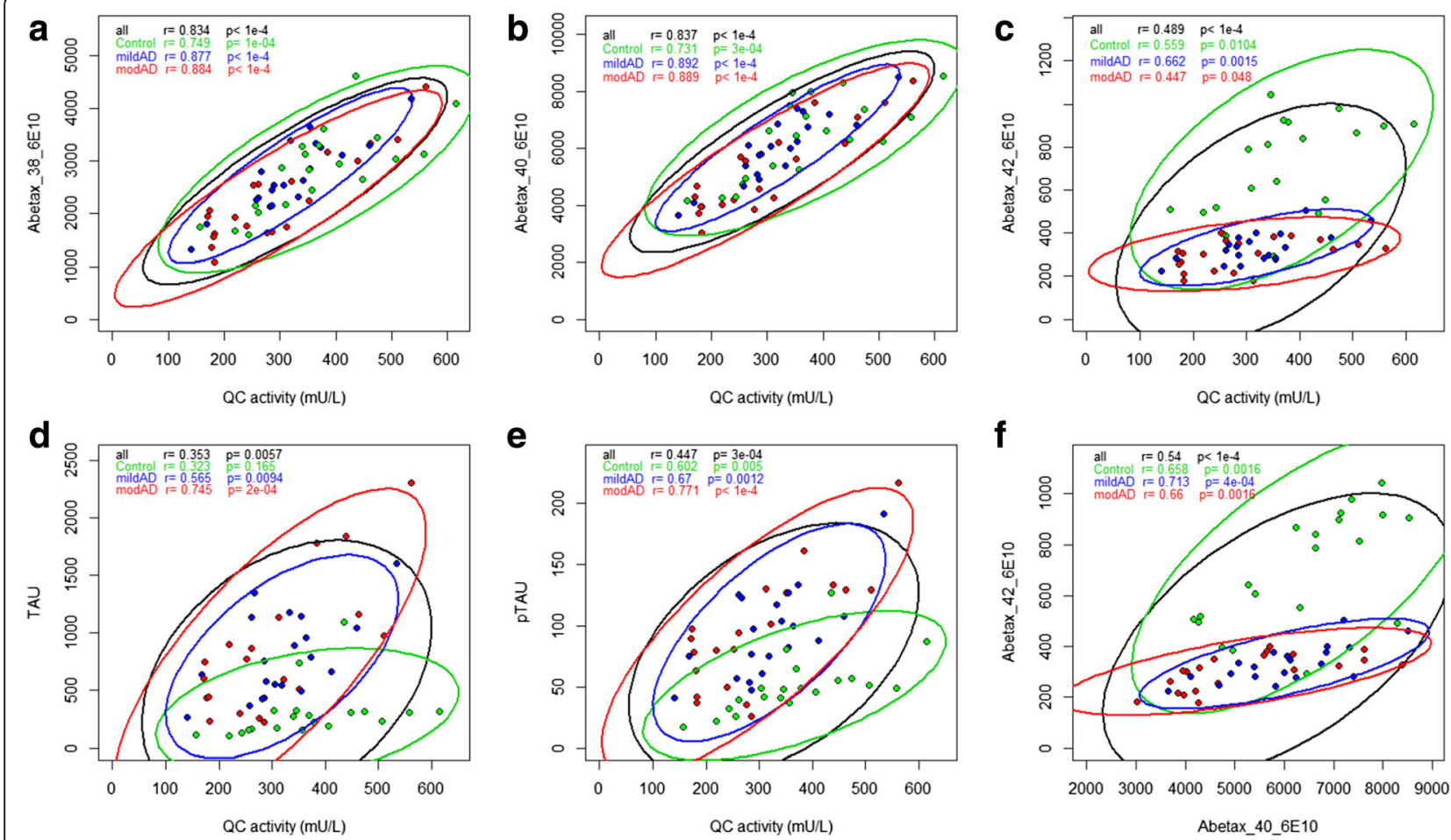

Fig. 3 Correlation of CSF QC activity (mU/L) with levels of $A \beta(\mathrm{pg} / \mathrm{ml})$ and Tau peptides $(\mathrm{pg} / \mathrm{ml})$ and $A \beta 40-A \beta 42$ correlation. a $A \beta 38$, b $A \beta 40, \mathbf{c}$ $A \beta 42$, d Tau, e ptau and $\mathbf{f} A \beta 40-A \beta 42$ correlation. Different diagnosis groups are colour coded (green: control, blue: mild AD, red: moderate AD). Pearson's correlation coefficient with $p$ values given for each parameter and group. Ellipses give $95 \% \mathrm{Cl}$ of the scatter for each group. Width and sloop of ellipses give a visual impression of strength of correlation: slim ellipses with sloops clearly different from 0 or 1 characterize high correlation of both parameters whereas wide ellipses with sloops narrow to 0 or 1 indicate less or no correlation. AD Alzheimer's disease, QC glutaminyl cyclase (Colour figure online)

In particular, $\mathrm{QC}$ activity correlated with angiogenesis mediators Flt1 $(r=0.711, p<0.0001)$, Tie2 $(r=0.602, p<$ $0.0001)$ and VEGF-D $(r=0.521, p<0.0001)$, as well as with soluble adhesion molecules ICAM-1 $(r=0.528)$ and VCAM-1 ( $r=0.501)$ (Fig. 5). No differences between the three groups were identified for the remaining inflammatory mediators (IL1- $\beta$, IL-6, IL-8, Eotaxin, Eotaxin-3, IP10, MDC, MIP- $1 \alpha$, MIP-1 $\beta$, TARC, SAA, PIGF) with the exception of IL-5, where a slight decrease was observed in the moderate AD group compared with control $(p=0.034)$. All other investigated inflammatory mediators (VEGF-C, IL- $1 \alpha$, IL-17a, TNF- $\beta$, IFN- $\gamma$, IL-10, IL-13, IL-2, IL-4, TNF- $\alpha$ ) were below the lower limit of detection in more than $1 / 3$ of samples across all three diagnostic groups and were thus not further evaluated (data not shown). QC activity did not correlate with MMSE scores or E4/E4 genotype (data not shown).

\section{Discussion}

In this study including 40 patients with probable $\mathrm{AD}$ and 20 controls with SMC, we find that QC activity shows a tendency towards decreased activity with progression of $\mathrm{AD}$ and that its addition to biomarkers tau and p-tau significantly increases the diagnostic power of these biomarkers. In $\mathrm{AD}$ individuals and controls, $\mathrm{QC}$ activity strongly correlates with levels of $A \beta 38$ and $A \beta 40$, as well as with levels of soluble ICAM-1, VCAM-1 and three angiogenesis mediators.

A mean QC activity of $366 \pm 116 \mathrm{mU} / \mathrm{L}$ (mean $\pm \mathrm{SD}$ ) was measured in CSF of SMC individuals, similar to the mean value of $376 \mathrm{mU} / \mathrm{L}$ reported by Lues et al. [28] using the same method in young (18-55 years old) healthy individuals. Although there was no significant difference between SMC and AD, we observed nominally decreased mean QC activities in mild $(319 \pm 89 \mathrm{mU} / \mathrm{L})$ and moderate $(297 \pm 120 \mathrm{mU} / \mathrm{L}) \mathrm{AD}$ compared with age-matched SMC. This tendency towards decreased $\mathrm{QC}$ activity in $\mathrm{AD}$ contrasts with the increased QC protein expression and $\mathrm{pE}-\mathrm{A} \beta$ levels reported in temporal and enthorinal cortex of AD patients [18], as well as with the increased QC mRNA levels reported in the brain [37] and blood [38] of AD patients. QC activity has not so far been measured in brain tissue, and the relation between expression level and activity of QC remains to be established. Defining the behaviour of $\mathrm{QC}$ activity with $\mathrm{AD}$ progression will require a more powered study as well as additional categories of disease severity ranging from MCI to severe AD. 


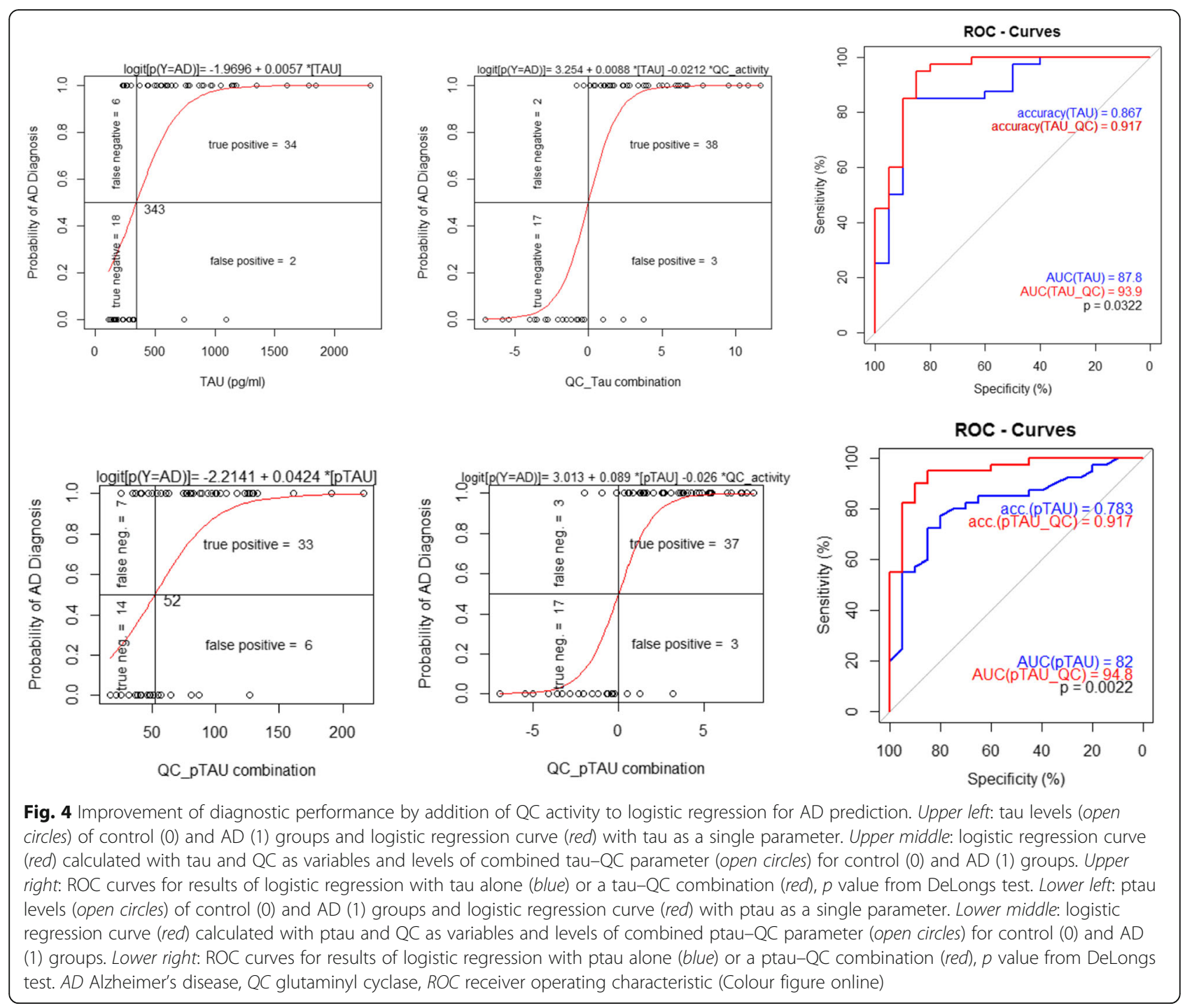

Conflicting results concerning $A \beta 38$ and $A \beta 40$ levels in CSF or plasma of AD patients are reported in the literature, ranging from decreased [39] or increased [40] levels in AD compared with controls to no changes between $\mathrm{AD}$ and controls [36, 41]. In our cohort, we observed a trend towards decreased $A \beta 38$ and $A \beta 40$ levels with $\mathrm{AD}$ progression, paralleling the tendency for decreased QC activity. In addition, QC activity showed very high positive correlation with $A \beta 38$ and $A \beta 40$, which together reflect the main portion of total $A \beta$ in the brain. This correlation is independent of diagnosis groups, which may point towards a higher level of interdependence or co-regulation of $A \beta 38, A \beta 40$ and $Q C$ activity. In the literature, the addition of $A \beta 38$ and $A \beta 40$ to core diagnostic biomarkers has been reported to improve discriminative power [36, 39-45]. In our cohort, we found that the addition of QC activity to diagnostic biomarkers tau and p-tau increased diagnostic power compared with the respective biomarkers alone. This finding may result from the high correlation and interdependence of $Q C$ activity with $A \beta 38$ and $A \beta 40$, further supporting the idea of a co-regulation of these parameters. It will be interesting to investigate, in clinical trials with $\mathrm{AD}$ patients, whether $\mathrm{QC}$ inhibition leads to reduced $A \beta 38$ and $A \beta 40$ levels, thus offering potential as pharmacodynamic markers of $Q C$ activity and surrogate markers of treatment response. Because $A \beta 38$ and $A \beta 40$ are included in multiplex $A \beta$ panels and can be easily assessed as part of routine AD workup in clinical trials, their assessment may offer practical advantages over QC activity measurement.

Using LC-MS/MS, QC substrates A $\beta 3-40, A \beta 11-40$ and $A \beta 11-42$ could be quantified along with full-length peptides $A \beta 1-40$ and $A \beta 1-42$. As for full-length $A \beta 40$, a trend towards decreased levels with $\mathrm{AD}$ progression was observed for $A \beta 3-40$, but not for $A \beta 11-40$. In contrast to 

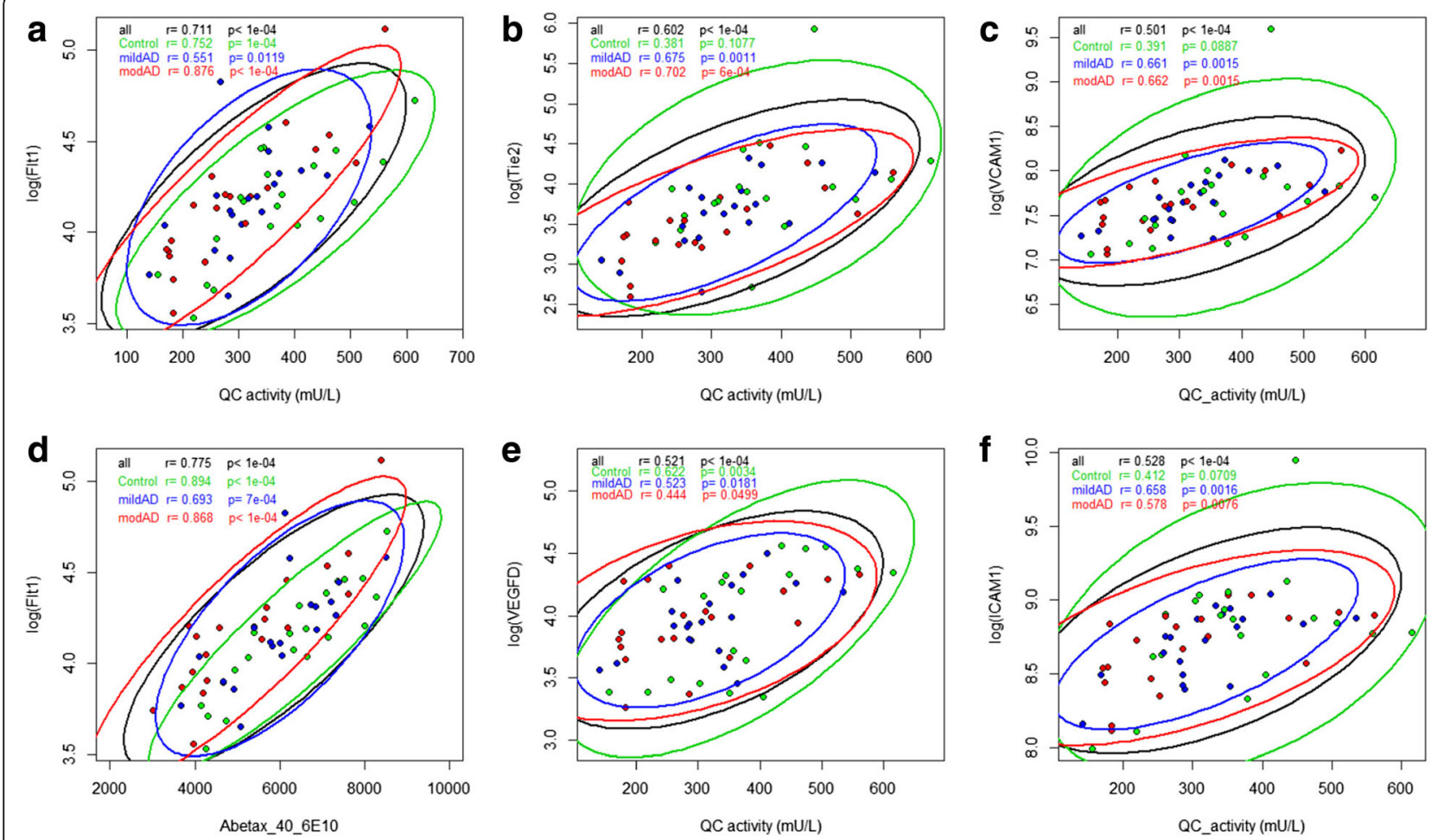

Fig. 5 Correlation of CSF QC activity ( $\mathrm{mU} / \mathrm{L}$ ) or A 40 with log-transformed levels of Flt1, Tie2, ICAM-1, VEGFD and VCAM-1. QC activity with a Flt1, b Tie2, c VCAM-1, e VEGFD and f ICAM-1. d A 440 with Flt1. Different diagnosis groups are colour coded (green: control, blue: mild AD, red: moderate AD). Pearson's correlation coefficient with $p$ values given for each parameter and group. Ellipses give $95 \% \mathrm{Cl}$ of the scatter for each group. Width and sloop of ellipses give a visual impression of strength of correlation: slim ellipses with sloops clearly different from 0 or 1 characterize high correlation of both parameters whereas wide ellipses with sloops narrow to 0 or 1 indicate less or no correlation. AD Alzheimer's disease, QC glutaminyl cyclase (Colour figure online)

its full-length counterpart $A \beta 42, A \beta 11-42$ levels were similar across all three diagnostic groups, and $A \beta 11-42$ and $A \beta 42$ levels did not correlate. This could indicate differences in solubility or proteolytic pathways involved in the generation of these peptides. Using ELISA on a similar sized cohort, Abraham et al. [46] found lower levels of $A \beta 11-40$ and $A \beta 11-42$ peptides in CSF of mild cognitive impairment (MCI) subjects compared with healthy controls. However, the levels of these peptides did not differ between $\mathrm{MCI}$ and $\mathrm{AD}$ subjects and $\mathrm{AD}$ and controls. Differences in group definition may explain the discrepancies with our findings.

In contrast to the high correlation with $A \beta 38$ and $A \beta 40$, only a moderate positive correlation between $\mathrm{QC}$ activity and core diagnostic biomarkers $\mathrm{A} \beta 42$, tau or ptau was observed. Correlations were greater when the three diagnosis groups were considered individually rather than pooled, further supporting a biological relevance of $\mathrm{QC}$ activity in $\mathrm{AD}$.

QC activity correlated with soluble adhesion molecules ICAM-1 and VCAM-1 and angiogenesis mediators including endothelial cell-specific receptors Tie-2 and Flt1 (a.k.a. VEGFR-1) as well as vascular endothelial growth factor VEGF-D. The latter correlation is of particular relevance, given the increasing evidence linking the VEGF pathway to AD pathology, and angiogenesis being hypothesized to play an active part in $\mathrm{AD}$ pathology [29]. A VEGF gene polymorphism has been associated with increased risk of $\mathrm{AD}[47,48]$, and $\mathrm{A} \beta$ was reported to block VEGFR-2-mediated signalling [49]. In mouse models of AD, VEGF was shown to increase neurogenesis and restore memory impairment [50-52]. From a biomarker perspective, decreased serum [53-55] and increased CSF [56] VEGF levels have been reported in AD. The correlation between QC activity and VEGF-D raises the possibility that QC inhibition influences this system. Further research is needed to determine whether this holds true and what the consequences may be.

\section{Conclusions}

We find that QC activity shows a tendency towards decrease with $\mathrm{AD}$ severity and correlates highly with some $A \beta$ peptides and mediators of angiogenesis. $A \beta 38, A \beta 40$, soluble ICAM-1 and VCAM-1, VEGF-D, Tie2 and Flt1 may serve as pharmacodynamic read-outs for QC inhibition and surrogate markers of treatment response, because their levels closely correlate with QC activity in $\mathrm{AD}$ 
patients. Moreover, the addition of these parameters to core diagnostic CSF biomarkers may improve diagnostic power, and be of specific interest in clinical cases with discordant imaging and core biochemical biomarker results.

\section{Additional files}

Additional file 1: Figure S2. showing method comparison for $A \beta s x-38$, $x-40$ and $x-42$ determination in CSF of AD patients and SMC: MSD A -6 E10 versus MSD A $\beta-4 G 8$ multiplex. Upper panels: scatter plots with regression line (Deming regression, blue solid) and line of identity (black dashed). Regression equation with 95\% confidence intervals of slope and intercept and coefficient of determination $\left(R^{2}\right)$ given at top of each figure. Lower panels: Bland-Altman plots with line of identity (solid, black), bias (solid, blue), limits of acceptance (dashed, blue) and $\pm 25 \%$ deviation between assays (dotted, black). AB38: regression line slope $(1.10(0.99,1.22))$ is slightly higher but not significantly different from 1, whereas intercept $(-561$ $(-878,-244) \mathrm{pg} / \mathrm{ml})$ is significantly below 0 . A 40 : slope $(1.02(0.89,1.14))$ and intercept $(-466(-1236,304) \mathrm{pg} / \mathrm{ml})$ are not significantly different from 1 or 0 , respectively. Regression line is parallel but slightly below the line of identity, resulting in slightly lower concentrations determined by the 4G8 assay (about $94 \%$ of 6 E10 in mean). In the Bland-Altman plot, there is no trend over the concentration range analysed and only four samples show more than $25 \%$ lower values compared with the $6 \mathrm{E} 10$ assay. A 342 : regression line slope of $0.92(0.84,0.99)$ is lower than 1 and the intercept of $55(19,92) \mathrm{pg} / \mathrm{ml}$ is significantly above 0 . Therefore, in the low concentration range $(<400 \mathrm{pg} / \mathrm{ml})$, higher values of $A \beta 42$ are determined with the $4 \mathrm{G} 8$ assay compared with the $6 \mathrm{E} 10$ assay. Because of the group difference, this affects most of the AD samples whereas for the control samples very similar values were determined with both assays (see Bland-Altman plot). (TIF 486 kb)

Additional file 2: Figure S3. showing method comparison for $A \beta$ determination in CSF of AD patients and SMC: LC-MS (relative quantification) versus MSD A $\beta$ multiplex. Upper panels: scatter plot with Deming regression lines. Lower panels, Bland-Altman plots for $A \beta 40$ assay comparison. For $A \beta 1-42$, only relative quantification was done with LC-MS. LC-MS determinations of $A \beta(1-40)$ and $A \beta(1-42)$ show correlation with both $A \beta$ multiplexes. Coefficients of determination were found to be slightly better with the $6 \mathrm{E} 10$ multiplex assays. For the AB40 assays, Deming regression lines show slope $<1$ and cross the lines of identity in the medium concentration range. Compared with the ELISAs, the LC-MS assay underestimates $A \beta 40$ in the low concentration range and overestimates it at high concentrations. Nevertheless, mean deviation is $<5 \%$ and there were only 5 out of 60 and 2 out of 60 measurements out of the $\pm 25 \%$ range compared with the $4 \mathrm{G} 8$ multiplex or $6 \mathrm{E} 10$ multiplex, respectively. (TIF $484 \mathrm{~kb}$ )

Additional file 3: Table S1. presenting a summary of the respective reference $A \beta$ peptides (sequence, molecular weight, charge state, selected SRM transitions and provider. $V^{*}=$ Val $(U 13 C 5 ; 15 \mathrm{~N})$ ), and Table S2 presenting relative response of endogenous $A \beta x-40$ peptides (analyte versus internal standard peak area ratios) extracted from a native CSF sample pool analysed over a storage period of 1 month at $-80^{\circ} \mathrm{C}(n=3)$. (DOCX $40 \mathrm{~kb}$ )

Additional file 4: Figure S1. showing LC-MS/MS chromatogram of endogenous full-length peptides $A \beta 1-40$ and $A \beta 1-42$ and N-terminally truncated peptides $A \beta 3-40, A \beta 11-40$ and $A \beta 11-42$ extracted from $100 \mu \mathrm{l}$ CSF. Aß3-42 was not detectable (see lane 5). (DOCX 85 kb)

\section{Abbreviations}

AD: Alzheimer's disease; ApoE: Apolipoprotein E; APP: Amyloid precursor protein; Aß: Amyloid beta; CSF: Cerebrospinal fluid; EEG: Electro-encephalography; MCl: Mild cognitive impairment; MMSE: Mini Mental State Evaluation; MRI: Magnetic resonance imaging; NIA-AA: National Institute on Aging-Alzheimer's Association; pE: Pyroglutamate; p-tau: Phosphorylated tau; QC: Glutaminyl cyclase; SMC: Subjective memory complaints; VEGF: Vascular endothelial growth factor

\section{Acknowledgements}

The authors thank Hans Heijst and Harry Twaalfhoven for technical advice.
Funding

CB is supported by a Swiss MS society grant.

\section{Availability of data and materials}

All data is available upon request to corresponding author.

\section{Authors' contributions}

$\mathrm{CB}$ analysed the data, designed and wrote the manuscript. TH designed the study, analysed the data and wrote the manuscript. AM performed the mass spectrometry measurements, wrote the corresponding methods section and critically reviewed the manuscript. SD performed biomarker measurements. MAK-S performed biomarker measurements. MO performed biomarker measurements. IL designed the study, analysed the data and critically reviewed the manuscript. PS critically reviewed the manuscript. CET designed the study, analysed the data and critically reviewed the manuscript. All authors read and approved the final manuscript.

\section{Competing interests}

$\mathrm{TH}, \mathrm{AM}$ and IL are employees of Probiodrug AG. CET received financial compensation from Probiodrug AG. The remaining authors declare that they have no competing interests.

\section{Consent for publication}

Not applicable.

\section{Ethics approval and consent to participate}

The present study was approved by the local ethical review board (Medisch ethische toetsings commissie, VUmc) and all subjects gave written informed consent for the use of their clinical data for research purposes.

\section{Publisher's Note}

Springer Nature remains neutral with regard to jurisdictional claims in published maps and institutional affiliations.

\section{Author details}

${ }^{1}$ Neurochemistry Lab and Biobank, Department of Clinical Chemistry, VU University Medical Centre Amsterdam, Amsterdam, The Netherlands. ${ }^{2}$ Probiodrug AG, Halle (Saale), Germany. ${ }^{3}$ Swiss BioQuant AG, Basel, Switzerland. ${ }^{4}$ Department of Neurology, Alzheimer Center, VU University Medical Centre Amsterdam, Amsterdam, The Netherlands.

Received: 26 January 2017 Accepted: 9 May 2017

Published online: 06 June 2017

\section{References}

1. Selkoe DJ, Hardy J. The amyloid hypothesis of Alzheimer's disease at 25 years. EMBO Mol Med. 2016:8:1-14.

2. Kummer MP, Heneka MT. Truncated and modified amyloid-beta species. Alzheimers Res Ther. 2014;6:28

3. Andrew RJ, Kellett KA, Thinakaran G, Hooper NM. A Greek Tragedy: the growing complexity of Alzheimer Amyloid precursor protein proteolysis. J Biol Chem. 2016;291(37):19235-44.

4. Cescato R, Dumermuth E, Spiess M, Paganetti PA. Increased generation of alternatively cleaved beta-amyloid peptides in cells expressing mutants of the amyloid precursor protein defective in endocytosis. J Neurochem. 2000;74:1131-9.

5. Takeda K, Araki W, Akiyama H, Tabira T. Amino-truncated amyloid $\beta$-peptide (A $\beta$ 5-40/42) produced from caspase-cleaved amyloid precursor protein is deposited in Alzheimer's disease. FASEB J. 2004;18(14):1755-7.

6. Saido TC, Yamao-Harigaya W, Iwatsubo T, Kawashima S. Amino- and carboxyl-terminal heterogeneity of beta-amyloid peptides deposited in human brain. Neurosci Lett. 1996;215:173-6.

7. He W, Barrow CJ. The A $\beta$ 3-pyroglutamyl and 11-pyroglutamyl peptides found in senile plaque have greater $\beta$-sheet forming and aggregation propensities in vitro than full-length $A \beta$. Biochemistry. 1999;38:10871-7

8. Schlenzig D, et al. Pyroglutamate formation influences solubility and amyloidogenicity of amyloid peptides. Biochemistry. 2009;48:7072-8.

9. Saido TC Alzheimer's disease as proteolytic disorders: anabolism and catabolism of beta-amyloid. Neurobiol Aging. 1998;19:569-75. 
10. Harigaya $Y$, et al. Amyloid beta protein starting pyroglutamate at position 3 is a major component of the amyloid deposits in the Alzheimer's disease brain. Biochem Biophys Res Commun. 2000;276:422-7.

11. Teng P, et al. HHS public access. Gynecol Oncol. 2015;136:554-61.

12. Mandler $M$, et al. Pyroglutamylated amyloid- $\beta$ is associated with hyperphosphorylated tau and severity of Alzheimer's disease. Acta Neuropathol. 2014;128:67-79.

13. Wirths $\mathrm{O}$, et al. Intraneuronal pyroglutamate-Abeta 3-42 triggers neurodegeneration and lethal neurological deficits in a transgenic mouse model. Acta Neuropathol. 2009;118:487-96.

14. Nussbaum JM, Seward ME, Bloom GS. Alzheimer disease: a tale of two prions. Prion. 2013;7:14-9.

15. Cynis H, Scheel E, Saido TC, Schilling S, Demuth HU. Amyloidogenic processing of amyloid precursor protein: Evidence of a pivotal role of glutaminyl cyclase in generation of pyroglutamate-modified amyloid- $\beta$. Biochemistry. 2008:47:7405-13.

16. Schilling $\mathrm{S}$, et al. Glutaminyl cyclase inhibition attenuates pyroglutamate Abeta and Alzheimer's disease-like pathology. Nat Med. 2008;14:1106-11.

17. Schilling S, Hoffmann T, Manhart S, Hoffmann M, Demuth HU. Glutaminyl cyclases unfold glutamyl cyclase activity under mild acid conditions. FEBS Lett. 2004:563:191-6.

18. Morawski M, et al. Glutaminyl cyclase in human cortex: correlation with (pGlu)-amyloid- $\beta$ load and cognitive decline in Alzheimer's disease. J Alzheimer's Dis. 2014;39:385-400

19. Cynis $\mathrm{H}$, et al. The isoenzyme of glutaminyl cyclase is an important regulator of monocyte infiltration under inflammatory conditions. EMBO Mol Med. 2011;3:545-58.

20. Oliveira EB, Gotschlich EC, Liu TY. Primary structure of human protein. J Bo Chem. 1979;254:489-502

21. Cynis $\mathrm{H}$, et al. Inhibition of glutaminyl cyclases alleviates CCL2-mediated inflammation of non-alcoholic fatty liver disease in mice. Int J Exp Pathol. 2013;94:217-25.

22. Eikelenboom P, Rozemuller AJ, Hoozemans JJ, Veerhuis R, van Gool WA. Neuroinflammation and Alzheimer disease: clinical and therapeutic implications. Alzheimer Dis Assoc Disord. 2000;14 Suppl 1:S54-61.

23. Rubio-perez JM, Morillas-ruiz JM. A review: inflammatory process in Alzheimer's disease, role of cytokines. Sci J. 2012;2012:756357.

24. Heppner FL, Ransohoff RM, Becher B. Immune attack: the role of inflammation in Alzheimer disease. Nat Rev Neurosci. 2015;16:358-72.

25. Kauwe JSK, et al. Genome-wide association study of CSF levels of 59 Alzheimer's disease candidate proteins: significant associations with proteins involved in amyloid processing and inflammation. PLoS Genet. 2014;10:e1004758

26. Sastre $\mathrm{M}$, et al. Nonsteroidal anti-inflammatory drugs and peroxisome proliferator-activated receptor-gamma agonists modulate immunostimulated processing of amyloid precursor protein through regulation of beta-secretase J Neurosci. 2003:23:9796-804.

27. Yan $Q$, et al. Anti-inflammatory drug therapy alters beta-amyloid processing and deposition in an animal model of Alzheimer's disease. J Neurosci. 2003;23:7504-9.

28. Lues I, et al. A phase 1 study to evaluate the safety and pharmacokinetics of PQ912, a glutaminyl cyclase inhibitor, in healthy subjects. Alzheimers Dement Transl Res Clin Interv. 2015;1:182-95.

29. Vagnucci AH, Li WW. Alzheimer's disease and angiogenesis. Lancet. 2003:361:605-8

30. McKhann GM, et al. The diagnosis of dementia due to Alzheimer's disease: recommendations from the National Institute on Aging-Alzheimer's Association workgroups on diagnostic guidelines for Alzheimer's disease. Alzheimers Dement. 2011;7:263-9.

31. Teunissen CE. A consensus protocol for the standardization of cerebrospinal fluid collection and biobanking; Neurology. 2009;73(22):1914-22.

32. Schilling $\mathrm{S}$, et al. Continuous spectrometric assays for glutaminyl cyclase activity. Anal Biochem. 2002;303:49-56.

33. Lame ME, Chambers EE, Blatnik M. Quantitation of amyloid beta peptides $A \beta(1-38), A \beta(1-40)$, and $A \beta(1-42)$ in human cerebrospinal fluid by ultraperformance liquid chromatography-tandem mass spectrometry. Anal Biochem. 2011;419:133-9.

34. Pannee J, et al. Round robin test on quantification of amyloid- $\beta$ 1-42 in cerebrospinal fluid by mass spectrometry. Alzheimers Dement. 2016;12:55-9.

35. Kuhlmann J, et al. CSF Aß1-42 —an excellent but complicated Alzheimer's biomarker-a route to standardisation. Clin Chim Acta. 2017;467:27-3.
36. Kleinschmidt $M$, et al. Characterizing aging, mild cognitive impairment, and dementia with blood-based biomarkers and neuropsychology. J Alzheimers Dis. 2015;50:111-26.

37. De Kimpe L, et al. Disturbed Ca2+ homeostasis increases glutaminyl cyclase expression; connecting two early pathogenic events in Alzheimer's disease in vitro. PLoS One. 2012;7:e44674.

38. Valenti MT, et al. Increased glutaminyl cyclase expression in peripheral blood of Alzheimer's disease patients. J Alzheimers Dis. 2013;34:263-71.

39. Baldeiras I, et al. Cerebrospinal fluid $A \beta 40$ is similarly reduced in patients with frontotemporal lobar degeneration and Alzheimer's disease. J Neurol Sci. 2015;358:308-16.

40. Dorey A, Perret-Liaudet A, Tholance Y, Fourier A, Quadrio I. Cerebrospinal fluid $A \beta 40$ improves the interpretation of $A \beta 42$ concentration for diagnosing Alzheimer's disease. Front Neurol. 2015;6:1-7.

41. Lewczuk $P$, et al. Cerebrospinal fluid $A \beta 42 / 40$ corresponds better than $A \beta 42$ to amyloid PET in Alzheimer's disease. J Alzheimers Dis. 2017;55(2):813-22.

42. Lewczuk $P$, et al. Neurochemical diagnosis of Alzheimer's dementia by CSF $A \beta 42, A \beta 42 / A \beta 40$ ratio and total tau. Neurobiol Aging. 2004;25:273-81.

43. Janelidze $S$, et al. CSF $A \beta 42 / A \beta 40$ and $A \beta 42 / A \beta 38$ ratios: better diagnostic markers of Alzheimer disease. Ann Clin Transl Neurol. 2016:3:154-65.

44. Kim HJ, et al. Elevation of the plasma Aß40-Aß42 ratio as a diagnostic marker of sporadic early-onset Alzheimer's disease. J Alzheimers Dis. 2015;48(4):1043-50

45. Blennow K, et al. Evolution of Abeta42 and Abeta40 levels and Abeta42 Abeta40 ratio in plasma during progression of Alzheimer's disease, a multicenter assessment. J Nutr Health Aging. 2009;13(3):205-8.

46. Abraham J-D, et al. Cerebrospinal A $111-x$ and $17-x$ levels as indicators of mild cognitive impairment and patients' stratification in Alzheimer's disease. Transl Psychiatry. 2013;3:e281.

47. Del Bo R, Ghezzi S, Scarpini E, Bresolin N, Comi GP. VEGF genetic variability is associated with increased risk of developing Alzheimer's disease. J Neurol Sci. 2009;283:66-8.

48. Chiappelli $M$, et al. VEGF gene and phenotype relation with Alzheimer's disease and mild cognitive impairment. Rejuvenation Res. 2006:9:485-93.

49. Patel NS, et al. Alzheimer's $\beta$-amyloid peptide blocks vascular endothelial growth factor mediated signaling via direct interaction with VEGFR-2. J Neurochem. 2010;112:66-76.

50. Religa P, et al. VEGF significantly restores impaired memory behavior in Alzheimer's mice by improvement of vascular survival. Sci Rep. 2013;3:2053.

51. Wang P, et al. VEGF-induced angiogenesis ameliorates the memory impairment in APP transgenic mouse model of Alzheimer's disease. Biochem Biophys Res Commun. 2011;411:620-6.

52. Herran E, et al. Enhanced hippocampal neurogenesis in APP-Ps1 mouse model of Alzheimer's disease after implantation of VEGF-loaded PLGA nanospheres. Curr Alzheimer Res. 2015;12(10):932-40.

53. Yu S, et al. Diagnostic utility of VEGF and soluble CD40L levels in serum of Alzheimer's patients. Clin Chim Acta. 2016:453:154-9.

54. Huang $L$, Jia J, Liu R. Decreased serum levels of the angiogenic factors VEGF and TGF- $\beta 1$ in Alzheimer's disease and amnestic mild cognitive impairment. Neurosci Lett. 2013;550:60-3.

55. Mateo I, et al. Low serum VEGF levels are associated with Alzheimer's disease. Acta Neurol Scand. 2007;116:56-8.

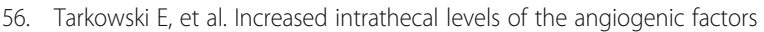
VEGF and TGF-beta in Alzheimer's disease and vascular dementia. Neurobiol Aging. 2002;23:237-43

\section{Submit your next manuscript to BioMed Central and we will help you at every step:}

- We accept pre-submission inquiries

- Our selector tool helps you to find the most relevant journal

- We provide round the clock customer support

- Convenient online submission

- Thorough peer review

- Inclusion in PubMed and all major indexing services

- Maximum visibility for your research

Submit your manuscript at www.biomedcentral.com/submit 